\title{
Anemomenotaktische Orientierung bei Skorpionen* (Chelicerata, Scorpiones)
}

\author{
K. Eduard Linsenmair \\ Zoologisches Institut der Universität Frankfurt a.M.
}

Eingegangen am 24. Juli 1968

\section{Anemomenotactic Orientation in Scorpions}

Summary. 1. Scorpions can orient menotactically to horizontal air currents (Fig. 1).

2. Changing the wind velocity from $0,05-0,1 \mathrm{~m} / \mathrm{sec}$ to $3-5 \mathrm{~m} / \mathrm{sec}$ has no influence on the menotactic angle kept by an anemomenotactic oriented scorpion (Fig. 2).

3. The receptors percieving the direction of air currents are the trichobothria.

4. Orientation to horizon landmarks, anemomenotactic and astromenotactic orientation does not exclude each other but complete themthelves mutually:

a) A scorpion orienting to horizon landmarks learns the corresponding anemomenotactic and astromenotactic angle (Fig. 4).

b) While orienting anemomenotactically (which is normally the main means of orientation when landmarks are absent) they continously learn new astromenotactical angles (Fig. 5), thus compensating for the movement of the moon or sun which can not be compensated otherwise.

c) Short calms and short changes of wind direction can be overcome by astrotaxis.

Bei Freilandversuchen zur Raumorientierung nordafrikanischer Wüstenskorpione der Gattungen Androctonus, Buthus, Buthotus, Scorpio u.a. war aufgefallen, daß die Versuchstiere (über 100 Individuen) auch nach Ausschaltung des Lichtsinnes eine Kompaßrichtung noch sicher einhalten konnten. Wie Laborversuche - vornehmlich mit Androctonus - zeigten, orientieren sie sich dabei nach horizontalen Luftströmungen (Abb. 1). Zu diesen können sie jeden beliebigen Winkel für mehrere Minuten, Stunden, manchmal sogar Tage einhalten. Art- oder populationsspezifische anemomenotaktische Vorzugswinkel ließen sich nicht feststellen; lediglich positive und negative Anemotaxis sind gegenüber menotaktischen Einstellungen selten.

* Ich danke der Deutschen Forschungsgemeinschaft für ihre Unterstützung, den Angehörigen der Elwerath-Wintershall, Tripolis für vielfältige Hilfe in Libyen und meiner Frau für wertvolle Hilfe bei den Freilandversuchen. 
Ein Einfluß absoluter oder - während des Laufens — wechselnder Windgeschwindigkeiten auf die einmal gewählte Vorzugsrichtung [wie beim Bachläufer Velia (Heran, 1962)] war bei Freiland- und Laborversuchen mit 26 Individuen in einem Bereich von etwa $0,05-0,1 \mathrm{~m} / \mathrm{sec}$ bis 3-5 m/sec nicht nachweisbar (Abb.2). Die untere Wahrnehmungsschwelle liegt zwischen $2-5 \mathrm{~cm} / \mathrm{sec}$, evt. auch noch etwas darunter. Die obere Grenze liegt bei solchen Geschwindigkeiten, gegen die die Tiere nicht mehr ankämpfen können.
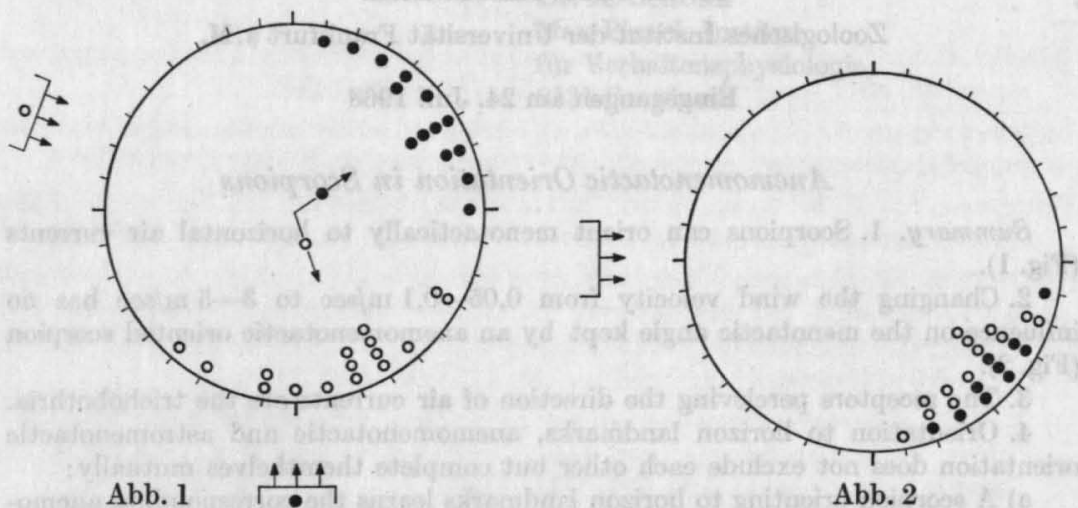

Abb. 1. Typische Reaktion eines sich anemomenotaktisch orientierenden Dickschwanzskorpions auf alternatives Umschalten zweier Windrichtungen. (Es werden Walzenventilatoren verwendet; die Windrichtung ist durch Pfeile außerhalb des Kreisdiagramms angegeben. Jedes Zeichen entspricht drei z.T. gemittelten Einzelläufen. Kreis oder Punkt = Laufrichtungen, die nach Einschalten des in der Zeichnung entsprechend gekennzeichneten Ventilators, gewählt werden)

Abb. 2. Schwankungen der Windgeschwindigkeiten haben keinen Einfluß auf die anemomenotaktisch eingehaltenen Vorzugsrichtung des Versuchstiers. Punkte = $2,5 \mathrm{~m} / \mathrm{sec}$; Kreise=Läufe bei $0,04-0,08 \mathrm{~m} / \mathrm{sec}$. (Jedes Zeichen entspricht zwei Läufen)

In ihren Trichobothrien haben die Skorpione Rezeptoren, die - nach den elektrophysiologischen Untersuchungen von HoFFManN (1967) ideal für die Richtungsfeststellung von Luftströmen geeignet sind. Ausschaltungsversuche an 14 Individuen zeigten, daß Skorpione ohne Trichobothrien zu einer Anemotaxis nach kontinuierlichen Luftströmungen nicht mehr fähig sind (Abb. 3). (Auch die Auslösung bestimmter Fluchtund Schreckverhaltensweisen und die spezifischer Beutefanghandlungen gegenüber fliegenden Insekten unterbleibt.) Auf kurze Windstöße hingegen reagieren Trichobothrienlose ebenso deutlich und gerichtet wie Intakte; die Rezeptoren, die sie zu dieser Leistung befähigen, sind noch nicht mit Sicherheit bekannt. 
Direkte Beobachtungen und Markierungsexperimente zeigten, daß Skorpione ihre Verstecke verlassen, um entweder deren nähere Umgebung nach Beute abzusuchen, wobei sie wieder zum Versteck zurückkehren, oder aber um gerichtet und endgültig von ihrem Versteck wegzuwandern. Horizontmarkenorientierung, Anemomenotaxis und Astromenotaxis ermöglichen diese gerichteten Wanderungen, wobei unter natürlichen Umständen (Nachtaktivität, Biotope mit praktisch dauerndem, relativ richtungskonstantem Wind) der Anemomenotaxis die größte Bedeutung zufällt.

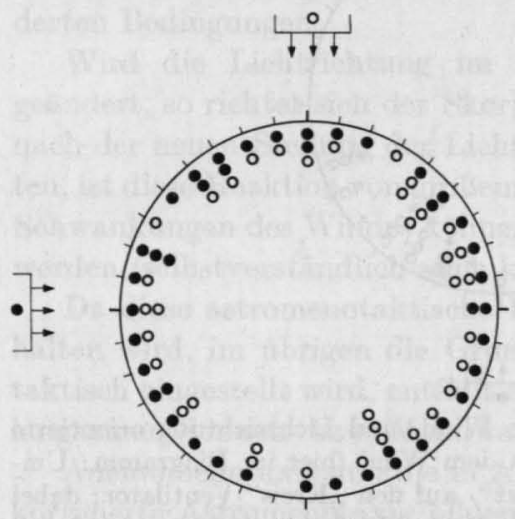

Abb. 3

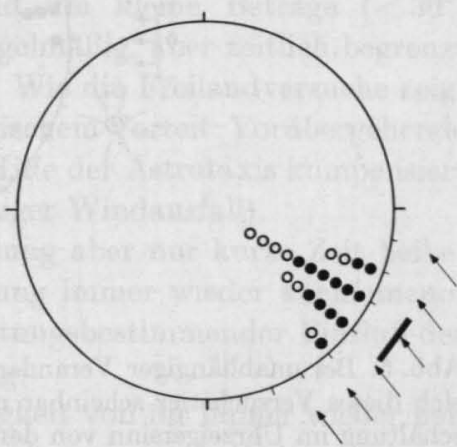

Abb. 4

Abb. 3. Skorpione - wie dieser Dickschwanzskorpion - ohne Trichobothrien sind zu einer Anemotaxis nicht mehr fähig. (Jedes Zeichen entspricht zwei Läufen)

Abb. 4. Dieses Versuchstier orientierte sich - im Freiland - nach einer Horizontmarke (deren Lage im Diagramm durch ein schwarzes Rechteck gekennzeichnet wurde, die Laufrichtungen sind durch Punkte wiedergegeben). Nach experimenteller Ausschaltung des Lichtsinnes wurde dieselbe Vorzugsrichtung (Kreise) mit Hilfe der Anemomenotaxis beibehalten. (Die Pfeile geben die Windrichtung an.) Geblendeten ist unter denselben Bedingungen und nach Entfernen der Trichobothrien eine Richtungsorientierung nicht mehr möglich. (Jedes Zeichen entspricht zwei

Läufen)

Wenn immer möglich orientieren sich die untersuchten Skorpione nach Horizontmarken, dabei wird aber die entsprechende Windrichtung (ebenso Mond- bzw. Sonnenstand) gelernt: Blendet man einen Skorpion, der sich eindeutig nach Horizontmarken und/oder einem Gestirn orientierte, so kann er - anemomenotaktisch - exakt dieselbe Kompaßrichtung einhalten (Abb. 4, Versuche mit 8 anderen Individuen erbrachten das gleiche Ergebnis).

Ist - wie bei allen im folgenden aufgeführten Versuchen - Orientierung nur nach einer Lichtquelle und dem Wind, nicht aber nach Horizontmarken, möglich, so erhält man bei einer unabhängigen Richtungsvariation der beiden Reize je nach der Größe der Winkelverschie- 
bung und je nachdem, ob die Versuche im Freiland oder im Labor (Lichtquelle : $100 \mathrm{~W}$ Leuchtstrahler in $2 \mathrm{~m}$ Entfernung vom Arenamp.) durchgeführt wurden, verschiedene Ergebnisse. Läßt man die Windrichtung konstant und ändert die Lichtrichtung um mehr als $60^{\circ}$, so richten sich die Versuchstiere nach den bisherigen Resultaten im Labor im Verhältnis 1:1 entweder vornehmlich nach der neuen Lichtrichtung oder der alten
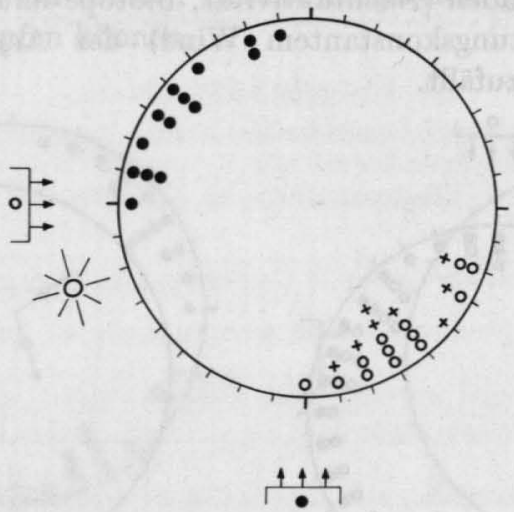

Abb. 5. Bei unabhängiger Veränderung von Wind- und Lichtrichtung orientierte sich dieses Versuchstier scheinbar nur nach dem Wind (hier im Diagramm: Umschaltung im Uhrzeigersinn von dem „Punkt"- auf den „Kreis"-Ventilator; dabei kommt es bei diesem Versuchstier zu einer Rechts-Linksumschaltung der Laufrichtungstendenz bezogen auf die Windrichtung. Auf diese - nicht sehr seltene Erscheinung - kann hier nicht eingegangen werden, die Stellung der Lampe ist jedenfalls nicht ihre Ursache.) Nach Ausschalten des Windes zeigte sich aber, daß die Lichtquelle beachtet und das neue Winkelverhältnis gelernt wurde: Der Skorpion behält - sich nach der Lichtquelle orientierend - die vorher anemomenotaktisch gesteuerte Vorzugsrichtung bei (Kreuze). (Jedes Zeichen entspricht zwei Läufen)

Windrichtung; selten kommt es auch zu deutlichen Kompromissen. Das neue Winkelverhältnis zwischen der ausschlaggebenden und der nicht ausschlaggebenden Reizquelle wird dabei nach jeder Änderung neu erlernt: Nach Ausschalten des ausschlaggebenden Reizes wird mit Hilfe der anderen Reizquelle die - relativ zu ihr — neue Richtung eingehalten (Abb. 5). Der Mistkäfer Geotrupes, das einzige Tier, bei dem bislang eine Anemomenotaxis nachgewiesen wurde (BIRUKow, 1958), entscheidet sich bei möglicher Orientierung nach Licht und Wind immer alternativ für eine Reizquelle. Die zweite bleibt anscheinend unbeachtet, weshalb auch eine relative Verschiebung zur orientierenden Reizquelle nicht erlernt wird.

Lichtrichtungsänderungen über $60^{\circ} \mathrm{im}$ Freiland führen bei den Skorpionen in der Regel zu keinen signifikanten Änderungen der Laufrichtungen, hier ist also der Wind der dominierende Reiz. 
Ein möglicher Grund für die unterschiedliche Reaktion im Freiland und Labor ist der, daß die Veränderung der Lichtrichtung im Freiland während des Laufens erfolgte, also für das Versuchstier — anhand seiner Stellung im Raum und des gleichbleibend gereizten Trichobothrienmusters - erkennbar gewesen sein könnte, wohingegen sie bei den Laborversuchen zwischen den Testläufen vorgenommen wurde, das Versuchstier also beim neuerlichen Einsetzen in die Arena plötzlich eine neue Situation vorfand. Für diese Deutung sprechen auch die ersten Befunde gerade begonnener Laborversuche unter entsprechend veränderten Bedingungen.

Wird die Lichtrichtung im Freiland um kleine Beträge $\left(<30^{\circ}\right)$ geändert, so richtet sich der Skorpion regelmäßig, aber zeitlich begrenzt nach der neuen Stellung der Lichtquelle. Wie die Freilandversuche zeigten, ist diese Reaktion von großem biologischem Vorteil: Vorübergehende Schwankungen des Windes können mit Hilfe der Astrotaxis kompensiert werden (selbstverständlich auch kurzzeitiger Windausfall).

Da diese astromenotaktische Einstellung aber nur kurze Zeit beibehalten wird, im übrigen die Grundrichtung immer wieder anemomenotaktisch eingestellt wird, entfällt ein richtungsbestimmender Einfluß der langsamen Sonnen- bzw. Mondwanderung.

Anemomenotaxis und die in Abhängigkeit von ihr immer wieder neu korrigierte Astromenotaxis bilden zusammen einen so leistungsfähigen Orientierungsmechanismus, daß eine - im Versuch mit Trichobothrienlosen nicht nachweisbare - zeitkompensierende astronomische Orientierung vorgetäuscht werden kann.

Die Versuchergebnisse sind der erste Nachweis:

1. einer biologischen Bedeutung der Trichobothrien und

2. der Anemomenotaxis bei Nicht-Insekten.

Vermutlich ist eine Anemotaxis bei Tieren, die in ihren Biotopansprüchen und ihrer Lebensweise den Wüstenskorpionen ähneln, nicht selten. Wir haben z.B. konkrete Anhaltspunkte, daß sich auch Walzenspinnen anemomenotaktisch orientieren können.

\section{Literatur}

BIRUkow, G.: Zur Funktion der Antennen beim Mistkäfer (Geotrupes silvaticus). Z. Tierpsychol. 15, 265-276 (1958).

Heran, H.: Anemotaxis und Fluchtorientierung des Bachläufers Velia caprai Tam.

Z. vergl. Physiol. 46, $129-149$ (1962).

Hoffmann, Сн.: Bau und Funktion der Trichobothrien von Euscorpius carpathicus L. Z. vergl. Physiol. 54, 290-352 (1958).

Dr. K. Eduard Linsenmair

Zoologisches Institut

6 Frankfurt a.M.

Siesmayerstr. 70 